\title{
BMJ Open Kukaa Salama (Staying Safe): study protocol for a pre/post-trial of an interactive mHealth intervention for increasing COVID-19 prevention practices with urban refugee youth in Kampala, Uganda
}

\author{
Carmen H Logie (1),${ }^{1,2,3}$ Moses Okumu, ${ }^{4}$ Isha Berry, ${ }^{5}$ Robert Hakiza, ${ }^{6}$ \\ Daniel Kibuuka Musoke, ${ }^{7}$ Peter Kyambadde, ${ }^{8}$ Simon Mwima, ${ }^{4,9}$ Richard T Lester, ${ }^{10}$ \\ Amaya G Perez-Brumer, ${ }^{11}$ Stefan Baral, ${ }^{12}$ Lawrence Mbuagbaw (1) ${ }^{13}$
}

To cite: Logie $\mathrm{CH}$, Okumu M, Berry I, et al. Kukaa Salama (Staying Safe): study protocol for a pre/post-trial of an interactive mHealth intervention for increasing COVID-19 prevention practices with urban refugee youth in Kampala, Uganda. BMJ Open 2021;11:e055530. doi:10.1136/ bmjopen-2021-055530

- Prepublication history for this paper is available online. To view these files, please visit the journal online (http://dx.doi org/10.1136/bmjopen-2021055530).

Received 15 July 2021 Accepted 28 October 2021

Check for updates

(C) Author(s) (or their employer(s)) 2021. Re-use permitted under CC BY-NC. No commercial re-use. See rights and permissions. Published by BMJ.

For numbered affiliations see end of article.

Correspondence to Professor Carmen H Logie; carmen.logie@utoronto.ca

\section{ABSTRACT}

Introduction With over 82.4 million forcibly displaced persons worldwide, there remains an urgent need to better describe culturally, contextually and age-tailored strategies for preventing COVID-19 in humanitarian contexts. Knowledge gaps are particularly pronounced for urban refugees who experience poverty, overcrowded living conditions and poor sanitation access that constrain the ability to practise COVID-19 mitigation strategies such as physical distancing and frequent hand washing. With over 1.4 million refugees, Uganda is sub-Saharan Africa's largest refugee hosting nation. More than 90000 of Uganda's refugees live in Kampala, most in informal settlements, and $27 \%$ are aged $15-24$ years old. There is an urgent need for tailored COVID-19 responses with urban refugee adolescents and youth. This study aims to evaluate the effectiveness of an 8-week interactive informational mobile health intervention on COVID-19 prevention practices among refugee and displaced youth aged 16-24 years in Kampala, Uganda.

Methods and analysis We will conduct a pre-test/posttest study nested within a larger cluster randomised trial. Approximately 385 youth participants will be enrolled and followed for 6 months. Data will be collected at three time points: before the intervention (time 1); immediately after the intervention (time 2) and at 16-week follow-up (time 3). The primary outcome (self-efficacy to practise COVID-19 prevention measures) and secondary outcomes (COVID-19 risk awareness, attitudes, norms and selfregulation practices; depression; sexual and reproductive health practices; food and water security; COVID-19 vaccine acceptability) will be evaluated using descriptive statistics and regression analyses.

Ethics and dissemination This study has been approved by the University of Toronto Research Ethics Board, the Mildmay Uganda Research Ethics Committee, and the Uganda National Council for Science \& Technology. The results will be published in peer-reviewed journals, and findings communicated through reports and conference presentations.
Strengths and limitations of this study

- The Kukaa Salama study is unique in exploring the use of mobile health (mHealth) technologies for improving COVID-19 prevention practices, including COVID-19 vaccine acceptance, among urban refugee and displaced youth in Kampala, Uganda.

- We use a pre-test/post-test longitudinal design to examine changes over time and assess if the 8week interactive informational mHealth intervention affects participants' self-efficacy to practise COVID-19 prevention measures.

- The primary study limitations are loss to follow-up and missing data points, as well as delays due to COVID-19.

- This research will inform us on the potential benefits of mHealth strategies in scaling up differentiated COVID-19 response efforts and messaging with urban refugee and displaced youth, and how this can be adapted for diverse contexts.

Trial registration number ClinicalTrials.gov Registry (NCT04631367).

\section{BACKGROUND}

This global public health emergency highlights the exclusion and multiple barriers to health care that are faced by migrants and refugees, among whom COVID-19 threatens to have rapid and devastating effects. - Global call to action for inclusion of migrants and refugees in the COVID-19 response (p1482) ${ }^{1}$ Refugee and other forcibly displaced persons experience healthcare barriers that require urgent attention in the COVID-19 pandemic to ensure that no one is left 
behind in public health responses. ${ }^{2}$ Poverty, overcrowded living conditions and poor sanitation elevate forcibly displaced persons' COVID-19 risks while limiting the ability to practise mitigation strategies (eg, physical distancing, hand washing). ${ }^{3-5}$ There is a pressing need to better understand culturally, contextually and agetailored strategies for preventing COVID-19 among the more than 82.4 million forcibly displaced persons worldwide. ${ }^{6}$ Although adolescents and youth comprise $42 \%$ of the world's forcibly displaced persons, ${ }^{6}$ they have been understudied in pandemics, and this is true for urban refugee youth who are often overlooked in research and programming due to a focus on settlement-based refugees. ${ }^{7-9}$ With over 1.4 million refugees, Uganda is subSaharan Africa's largest refugee hosting nation. ${ }^{10}$ More than 90000 of Uganda's refugees live in Kampala, most in informal settlements, and 27\% are aged 15-24 years old. ${ }^{10}$ Language and communication barriers have been identified as barriers to accessing COVID-19 information among urban refugees at large in Kampala, ${ }^{11}$ and knowledge gaps persist regarding efficacious strategies to increase knowledge and uptake of COVID-19 prevention practices among urban refugee youth.

The WHO's recommended COVID-19 mitigation practices include hand hygiene (washing hands regularly and thoroughly with soap and water, avoiding touching mouth, eyes or nose); respiratory hygiene (covering mouth or nose when coughing or sneezing, then washing hands); physical distancing (maintaining at least 1-metre distance with others; in densely populated contexts this may involve household or community shielding); and wearing a mask (including appropriate storage and daily cleaning of cloth masks). ${ }^{12}$ These calls for physical distancing, hand and respiratory hygiene, and mask wearing with daily cleaning practices may not be realised among those living in informal settlements due to overcrowded living conditions, poverty, and poor access to water and sanitation. ${ }^{2313}$

There are knowledge gaps regarding some of these recommended hygiene practices with adolescents and youth in humanitarian contexts. For instance, we identified no respiratory hygiene intervention studies in humanitarian contexts. ${ }^{14-16}$ Humanitarian context hand hygiene studies report that information is necessary-but not sufficient-to motivate hand hygiene ${ }^{17-19}$ and call for behaviour change strategies. ${ }^{18}$ No adolescent/youth hand hygiene studies were located in humanitarian contexts. A study with internally displaced children in Iraq identified hand hygiene determinants included hygiene promotion, social norms and motivational drivers. ${ }^{19} \mathrm{~A}$ hand hygiene study with displaced adults in the Democratic Republic of Congo highlighted the role of emotional and social motivators and a need for innovation. ${ }^{20}$ Physical distancing is not feasible in crowded settlements, ${ }^{21}$ yet limited research has assessed strategies to promote alternatives such as household or community shielding. ${ }^{3} 22$

Our study applies behavioural science to mobile health (mHealth) to increase COVID-19 preventive practice uptake with urban refugee youth. As Michie described, 'behavioural science must be at the heart of the public health response' ( $p 1$ ) for COVID-19 mitigation. ${ }^{23}$ We follow the Risk, Attitude, Norms, Ability, Self-regulation (RANAS) approach to systematic behaviour change for Water and Sanitation and Hygiene (WASH) grounded in three contexts (social, physical, personal) and five behavioural factors: risk (eg, risk and vulnerability awareness), attitude (eg, costs/benefits of the behaviour), norms (eg, behaviours others adopt), ability (eg, efficacy, confidence), and self-regulation (eg, action and barrier planning). ${ }^{24}{ }^{25}$ RANAS - a widely used framework for WASH interventions-provides behavioural change techniques to address behavioural factors. ${ }^{26-29}$

mHealth is a cost-effective health information delivery approach that is aligned with how youth learn and socialise, and important for physical distancing. Short messaging services (SMS), or text messages, have been used to disseminate hygiene messaging in humanitarian contexts, ${ }^{18}$ and by the Office of the United Nations High Commissioner for Refugees (UNHCR) for COVID-19 symptom reporting in Kenya. ${ }^{5}$ Similarly, Singapore is using WhatsApp ${ }^{30}$ to share COVID-19 updates with healthcare providers. ${ }^{31}$ In particular, interactive mHealth interventions including multiple forms of media and engagement including SMS, WhatsApp and group photo sharing could be more effective than one-way messages/ reminders for changing behaviour. ${ }^{32-36}$ The potential to implement mHealth solutions at scale is high given that most urban refugees in Uganda have access to mobile phones. ${ }^{37}$ Yet, in 2021, the use of mHealth for RANASbased behaviour change remains understudied among urban refugee youth.

Kukaa Salama, roughly translated from Swahili for 'staying safe', aims to address these knowledge gaps by evaluating an interactive informational mHealth intervention on COVID-19 prevention practices. This study is nested within a cluster randomised trial on HIV selftesting for which the primary outcomes are HIV testing frequency and status knowledge (Tushirikiane; ClinicalTrials.gov registration NCT04504097), which recently completed data collection in Kampala, Uganda. ${ }^{38}$ Findings from this study can be used to inform local and global response efforts with new knowledge of mHealth approaches for COVID-19 prevention with urban refugee youth in humanitarian contexts.

\section{METHODS}

\section{Study aim and objectives}

The overarching goal of this study is to evaluate the effectiveness of an 8-week interactive informational mHealth intervention on increasing COVID-19 prevention practices among refugee and displaced youth aged 16-24 years in Kampala, Uganda. The primary objective is to evaluate the effectiveness of the intervention on participants' selfefficacy (eg, ability, confidence and adherence) to practise hand and respiratory hygiene COVID-19 prevention 
measures. Secondary objectives include examining the impact of the intervention on (1) COVID-19 risk awareness; (2) attitudes towards COVID-19; (3) perceived COVID-19 norms; (4) COVID-19 self-regulation practices; (5) depression; (6) sexual and reproductive health practices; (7) food and water security perceptions; and (8) COVID-19 vaccine acceptability.

\section{Study design}

To evaluate the intervention's effectiveness, we are conducting a single arm, pre-test/post-test trial design. A control group design is intentionally not used based on recommendations regarding ethical concerns over the potential withholding of any public health intervention benefits from a vulnerable group (ie, refugee youth living in informal settlements) in the midst of a pandemic. ${ }^{39}$ Data will be collected at three time points: at enrolment before the intervention (time 1); immediately after the completion of the mHealth intervention at 8 weeks (time 2) and at a 16-week follow-up survey (time 3). Participant demographic data will be linked from the Tushirikiane cohort, which collected baseline data in February 2020.

\section{Study setting}

This trial is being conducted in five informal settlements in Kampala, Uganda (Kabalagala, Kansanga, Katwe, Nsyamba, Rubaga). Enrolment and clinical activities are based at the Young African Refugees for Integral Development (YARID) centre, a youth-focused communitybased non-governmental organisation that implements economic empowerment programmes for refugee youth. Full details regarding the trial site geography and population have been described elsewhere. ${ }^{38}$

\section{Participants and recruitment}

All participants enrolled in the Tushirikiane trial are eligible for the Kukaa Salama substudy. Tushirikiane participants were recruited using purposive methods, including peer-driven recruitment with the support of 12 peer navigators. Peer navigators are study staff who identify as refugees or displaced persons, aged 18-24 years, who have been engaged to help with recruitment and retention, as well as providing feedback on study designs and surveys. Inclusion criteria for participants into the Kukaa Salama study include: (1) being a Tushirikiane participant, (2) living in one of the five informal settlement sites (Kabalanga, Kansanga, Katwe, Nsambya or Rubaga); (3) identifying as a refugee/displaced person or have been born to refugee/displaced persons; (4) aged 16-24 years; (5) speaking one of the study languages (English, French, Kinyarwanda/Kirundi, Luganda or Swahili); and (6) owning or having access to a mobile phone for the duration of the study.

Participants are informed of the Kukaa Salama substudy at a clinical visit after enrolment into the original trial. We obtain separate written informed consent for participation in Kukaa Salama. Participants are free to refuse participation or withdraw from Kukaa Salama while remaining in Tushirikiane. However, Tushirikiane participants who withdraw or are lost to follow-up are automatically withdrawn from the Kukaa Salama substudy. Community collaborators and peer navigators will facilitate participant retention using multiple study reminder strategies to maintain engagement.

\section{Patient and public involvement}

This study protocol was developed after the completion of a formative qualitative research phase. During this formative research, we engaged in in-depth interviews with youth participants from the Tushirikiane study as well as local key informants, who are professionals in various roles supporting health and well-being of refugee youth in Uganda, to identify key priorities and preferences. This qualitative component informed the development of the intervention and key themes for the COVID-19 prevention messaging; therefore, this study directly responds to refugee youth identified needs. Additionally, this research is being conducted in collaboration with YARID, and study collaborators have been involved in all aspects of the research cycle, from development of the study question to implementation. In particular, the YARID peer navigators have been consulted regarding the study design and outcomes, supported participant recruitment and engagement, and have pilot-tested all study instruments to assess acceptability for the study population. All peer navigators' feedback has been integrated into the final intervention and study instruments, with modifications made to ensure appropriate delivery. The key study findings will be disseminated to participants and community members through an integrated knowledge translation approach, including infographics and community meetings developed in collaboration with local partners.

\section{Intervention description}

This is a pre-test/post-test trial, therefore all participants will receive the Kukaa Salama intervention. Kukaa Salama is an 8-week mHealth programme of COVID-19 prevention messaging, which includes sending weekly informational SMS and facilitated secure group interactions to share multimedia images. The intervention is hosted on a web-based SMS platform developed by WelTel ${ }^{35} 3640$ and accompanied by moderated group interactions and photo sharing using WhatsApp. WelTel is a non-profit agency developing the mHealth intervention, in which participants receive weekly supportive bidirectional text messages. WhatsApp is a no cost mobile phone application that allows users to share SMS, photos, videos and voice messages with end-to-end encryption; requires minimal data and is widely used to share health information. ${ }^{32} 3341-44$ The intervention involves three linked mHealth components and a prevention parcel, which are described below and summarised in figure 1 .

\section{Check-in messaging}

Each week, participants will receive a message asking how they are doing and are requested to reply 'fine' 
Time 1

(Pre-intervention)

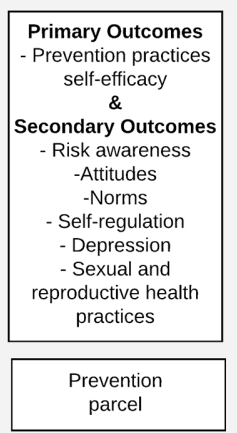

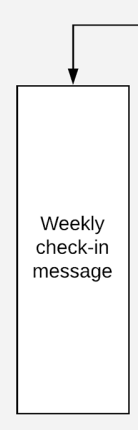

Time 2

(Post-intervention)

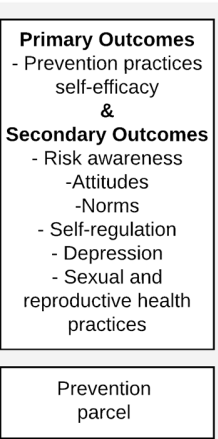

Time 3

(Follow-up survey)

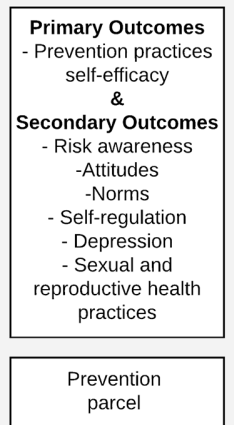

Figure 1 Study design for Kukaa Salama, a pre-test/post-test trial of an interactive and informational mobile health (mHealth) strategy among urban refugee and displaced youth in Kamala, Uganda.

or 'not fine'; those responding 'not fine' will be contacted for support by a peer navigator. The WelTel system will manage the SMS intervention on a structured mobile phone platform (all SMS interactions are logged).

\section{COVID-19 prevention messaging}

Participants will also receive a weekly themed COVID-19 informational SMS and an accompanying question to enhance engagement. Content topics address the themes identified during our formative qualitative research phase and apply behaviour change techniques (BCT) approaches following the RANAS ${ }^{25}$ framework. Weekly themed topics include information on COVID-19 symptoms and transmission; encouragement on the use of hand washing, respiratory hygiene, and face masks; COVID-19 vaccines and testing; and stigma and recovery (table 1). All messages will be translated into the five study languages; in the pre-intervention survey, participants indicate their language preference for receiving health-related SMS, and the preferred language will be programmed for each participant. The peer navigators will review the responses to the engagement question and the top response will be sent out to all participants to incentivise engagement. Participants can also respond to the SMS with any questions about COVID-19, and the study team will respond with further information and/or additional resources.

\section{COVID-19 multimedia sharing}

Finally, within the peer navigator-facilitated secure and encrypted WhatsApp groups, participants are able to share multimedia images related to the weekly theme. These can include photos, memes, GIFs and other multimedia options; this provides an opportunity for participants to display their application and practice of the weekly COVID-19 prevention topics in their daily lives. This aligns with calls for hygiene communication in humanitarian contexts to include information and images. ${ }^{18}$

\section{COVID-19 prevention parcel}

At each survey time point, participants will also be offered the opportunity to pick up a parcel that contains a face mask, bar of soap and a small parcel of

\begin{tabular}{|c|c|}
\hline Scenario & Description \\
\hline Mental health & $\begin{array}{l}\text { Explores strategies for managing stress and supporting mental health and well-being during } \\
\text { COVID-19, including how peers can support each other. }\end{array}$ \\
\hline Vaccine hesitancy & Presents the importance of taking a COVID-19 vaccine to protect oneself and their community. \\
\hline Hand washing & $\begin{array}{l}\text { Explains the importance of hand washing for COVID-19 prevention, and provides tips on when one } \\
\text { should wash their hands. }\end{array}$ \\
\hline Mask wearing & $\begin{array}{l}\text { Explains the importance of wearing masks to reduce COVID-19 transmission, and provides tips on } \\
\text { when one should wear a mask. }\end{array}$ \\
\hline Economic stressors & $\begin{array}{l}\text { Acknowledges the personal and community hardships caused by COVID-19, and elicits peer } \\
\text { support for overcoming hardship. }\end{array}$ \\
\hline Symptoms and testing & $\begin{array}{l}\text { Provides a reminder of key COVID-19 symptoms and addresses the importance of COVID-19 } \\
\text { testing as a pathway to care. }\end{array}$ \\
\hline Stigma and recovery & $\begin{array}{l}\text { Addresses stigma associated with COVID-19 and COVID-19 recovery, and encourages peer } \\
\text { support during and after recovery. }\end{array}$ \\
\hline Recap week & Reviews the key themes and encourages peer and community support. \\
\hline
\end{tabular}


food to support the prevention messaging and address food insecurity.

\section{Outcomes}

The primary outcome measured in this trial is:

Changes in COVID-19 prevention practices, assessed by asking participants to report on their self-efficacy (ie, ability, confidence and adherence) to practise hand and respiratory hygiene (eg, hand washing with soap, face mask usage) and physical distancing. Questions use the RANAS framework questions ${ }^{25} 2745$ applied to these preventive practices. This measure is assessed at all three study time points (pre-intervention (time 1), postintervention (time 2) and follow-up (time 3)).

Secondary outcomes are below; secondary outcomes 1-4 follow the RANAS questionnaire items ${ }^{25} 2745$ adapted for COVID-19 preventive practices:

1. Changes in COVID-19 risk awareness, assessed by asking participants to report on their perceived risk and vulnerabilities to COVID-19 as well as their knowledge of symptoms and severity (time 1 , time 2 , time 3 ).

2. Changes in attitude towards COVID-19, assessed by asking participants to report on their attitude (ie, feelings, costs/benefits) towards COVID-19 prevention practices as well as towards COVID-19 testing and potential vaccines (time 1 , time 2 , time 3 ).

3. Changes in COVID-19 norms, assessed by asking participants to report on the perceived behaviours approved by others (ie, social pressures) towards COVID-19 prevention practices, transmission and stigma (time 1 , time 2 , time 3 ).

4. Changes in COVID-19 self-regulation, assessed by asking participants about their action plan for implementing COVID-19 prevention practices (time 1 , time 2, time $3)$.

5. Changes in depression, assessed using the Patient Health Questionnaire-9 item ${ }^{46}$ (time 1, time 2, time 3). Scores range from 0 to 27; higher scores mean a worse outcome.

6. Changes in sexual and reproductive health practices, assessed by asking participants to report on personal experiences (eg, intimate partner violence) as well as perceived changes in the community (eg, violence, access to sexual and reproductive health services) (time 1 , time 2 , time 3 ).

7. Changes in food and water insecurity, which is assessed by asking participants to report on frequency of insufficient food (ie, going to bed hungry) and inadequate clean water (time 1 , time 2 , time 3 ).

\section{Sample size and power}

We estimate that a sample size of 52 participants (104 data points) is required to detect an effect size of 0.4 between pairs with a power of $80 \%$ and type 1 error rate of $5 \%$, and assuming a correlation between pre-test/posttest responses of 0.5 . For a correlation as low as 0.1 , we estimate that 91 participants will be required; and if the effect size is larger, we will require fewer participants.
Based on current participant retention rates, we anticipate that at least $85 \%(\mathrm{n}=340)$ of the Tushirikiane cohort $(\mathrm{n}=404)$ will participate in Kukaa Salama. This will give us sufficient power for conducting this analysis, as well as for covariate adjustment.

\section{Data collection and management}

Participant outcome data will be collected at three time points (pre-intervention, time 1; post-intervention, time 2; follow-up, time 3) using structured questionnaires administered by trained research assistants. Interviews will be conducted in all study languages and data will be collected in person or by mobile telephone, depending on local COVID-19 guidelines. All data will be recorded on tablets using SurveyCTO (Dobility, Cambridge, USA). Data collection tools include branching logic for efficiency and have range and consistency checks built-in to provide immediate feedback to research assistants regarding errors and inconsistencies. Tablet-based data collected will be automatically encrypted and uploaded to a password-protected project team server on a daily basis using a Secure Sockets Layer (SSL) certificate. To maintain confidentiality, all participants have been given a unique case ID, and no personal identifying information will be stored with the study data.

\section{Data analysis}

All analyses and reporting will be conducted following the Transparent Reporting of Evaluations with Nonrandomized Designs (TREND) checklist. ${ }^{47}$ Descriptive analyses of sociodemographic variables will be conducted to characterise the participant sample using counts or means and SDs or medians and IQRs, as appropriate. Outcome scale items will be summed to calculate overall and subscale scores, and novel scales will be assessed for reliability (ie, Cronbach's alpha). To assess pre/postoutcome differences in RANAS scores, we will use linear or logistic generalised estimating equation regression models, depending on which outcome is being evaluated, with an exchangeable correlation matrix to account for clustering by participant. To assess the moderating effect of engagement with the intervention, we will examine interactions between mean outcome pre/postscore changes and intervention usage (eg, WelTel data regarding frequency of interaction, number of days used, length of time, etc). We will conduct adjusted analyses to examine the role of covariates on the relative effect. Covariates (eg, age, gender) will be entered as a block. The level of significance will be set at alpha $=0.05$, and we will report ORs or mean differences as appropriate, and corresponding $95 \%$ CIs and $p$ values. Given this pre/posttrial is of short duration with minimal risks, a data monitoring committee was not deemed necessary.

\section{DISCUSSION}

There are knowledge gaps regarding efficacious strategies to increase COVID-19 preventive practices with urban 
refugee youth-despite calls for inclusion of migrants and refugees in COVID-19 responses. ${ }^{1}$ The current study aims to address this knowledge gap by conducting an 8-week interactive and informational mHealth intervention to improve COVID-19 prevention practices among refugee youth in Kampala, Uganda. Study strengths are: the intervention is included within an existing communitybased research cohort study that leverages trained peer navigators and engaged community and government collaborators; the design and foci were informed by qualitative research with urban refugee youth and key informants to address identified needs; and alignment with the well-established RANAS framework. ${ }^{25}$ There are also study limitations due to the pre/post-test design with no control group; other study designs such as stepped wedge could be implemented in future iterations. In addition, given the COVID-19 pandemic, we have seen higher rates of loss to follow-up as participants leave urban areas and return to their country of origin or to settlements/camps. Political instability and interruptions to internet access in Uganda in early 2021 also delayed study implementation.

This study will provide effectiveness data for a nonpharmaceutical intervention that is low cost, scalable, contextually specific and rooted in behavioural science, which is essential with limited availability of COVID-19 vaccines in Uganda and other sub-Saharan African contexts. If findings indicate that the intervention increases COVID-19 prevention practices in this population, there is scope to scale up this intervention for other urban refugee populations in Uganda, and it can be adapted for other informal settlement populations as well as non-urban settlement-based refugees.

\section{ETHICS AND DISSEMINATION \\ Ethical approval}

The Kukaa Salama intervention study was approved as an amendment to the Tushirikiane trial by the University of Toronto Research Ethics Board (REB) (reference 37496); the Mildmay Uganda Research Ethics Committee (reference 0806-2019); and the Uganda National Council for Science \& Technology (reference HS 2716). All participants have provided written informed consent for inclusion in this study.

\section{Dissemination plan}

Irrespective of study findings, results will be published in peer-reviewed scientific journals following international authorship guidelines, and will be presented to academics and researchers at key scientific conferences. Study results will also be shared as executive summaries, reports and technical policy briefs with national and international collaborating organisations, including our collaborators, the Uganda Ministry of Health and UNHCR. Engaging pictorial research summaries with highlights of study findings in all five languages will also be shared with participants and study collaborators.
Box 1 Items from the US National Institutes of Health Trial Registry

Data category information

Primary registry and trial identifying number: ClinicalTrials.gov NCT04631367.

Date of registration: 13 November 2020.

Source(s) of monetary support: International Development Research Center.

Primary sponsor: University of Toronto.

Contact for public and scientific queries: Carmen Logie, PhD (carmen.logie@utoronto.ca).

Public and scientific title: mHealth Intervention for Increasing COVID-19 Prevention Practices With Urban Refugee and Displaced Youth in Uganda.

Countries of recruitment: Uganda.

Health condition(s) or problem(s) studied: COVID-19 prevention practices.

Intervention(s): Kukaa Salama: mHealth intervention; face mask+soap.

Key inclusion criteria: enrolled within the Tushirikiane HIV self-testing cluster randomised trial; live in one of the five slum/informal settlement sites (Kabalanga, Kasanga, Katwe, Nsambya, Rubaga); identify as a refugee/displaced person or have refugee parents; age 16-24 years; speak English, Luganda, French, Swahili or Kinyarwanda; own or have access to a mobile phone for the duration of the study.

Study type: interventional; single group assignment pre/post-trial; primary purpose: behavioural change.

Date of first enrolment: June 2021.

Target sample size: 404.

Primary outcome(s): changes in COVID-19 prevention practices assessed by asking participants to report on their self-efficacy to practise hand and respiratory hygiene and physical distancing.

Key secondary outcomes: (1) changes in COVID-19 risk awareness; (2) changes in attitude towards COVID-19: (3) changes in COVID-19 norms; (4) changes in COVID-19 self-regulation; (5) changes in depression; (6) changes in sexual and reproductive health practices; (7) changes in food and water insecurity; (8) changes in COVID-19 vaccine acceptability.

mHealth, mobile health.

\section{Trial status}

The Kukaa Salama study was launched in April 2021 with pre-intervention data collection. The intervention is currently underway. We anticipate data collection postintervention to be conducted in August 2021, and the final follow-up survey to be conducted in October 2021. Any important protocol modifications will be included as amendments in REB and updated on the ClinicalTrials. gov registry, as and when needed. Box 1 details the information on the ClinicalTrials.gov registry.

Author affiliations

${ }^{1}$ Factor-Inwentash Faculty of Social Work, University of Toronto, Toronto, Ontario,

Canada

${ }^{2}$ Women's College Research Institute, Women's College Hospital, Toronto, Ontario, Canada

${ }^{3}$ Centre for Gender \& Sexual Health Equity, Vancouver, BC, Canada

${ }^{4}$ School of Social Work, University of Illinois at Urbana-Champaign, Urbana, Illinois, USA

${ }^{5}$ Dalla Lana School of Public Health, University of Toronto, Toronto, Ontario, Canada 
${ }^{6}$ Young African Refugees for Integral Development, Kampala, Uganda

${ }^{7}$ International Research Consortium, Kampala, Uganda

${ }^{8}$ National AIDS Control Program, Ministry of Health, Kampala, Uganda

${ }^{9}$ Most At Risk Population Initiative (MARPI) Clinic, Mulago Hospital, Kampala, Uganda

${ }^{10}$ Department of Medicine, University of British Columbia, Vancouver, British

Columbia, Canada

${ }^{11}$ University of Toronto, Toronto, Ontario, Canada

${ }^{12}$ Johns Hopkins Bloomberg School of Public Health, Johns Hopkins University,

Baltimore, Maryland, USA

${ }^{13}$ Department of Clinical Epidemiology and Biostatistics, McMaster University, Hamilton, Ontario, Canada

Acknowledgements We would like to acknowledge the support and contributions of: Young African Refugees for Integral Development (YARID), Uganda Ministry of Health, Uganda National AIDS Control Program, Dr Gabby Serafini (WelTel), Interaid Uganda, Mildmay Uganda, Organization for Gender Empowerment and Rights Advocacy (OGERA Uganda), Most At Risk Population Initiative (MARPI), Uganda Office of the Prime Minister Department of Refugees, peer navigators (Gabriella Nzulungi; Bibishe Hakiza Muruta; Sabrina Gamwanya; Hillary Nuwamanya; Justin Paluku; Bella Nshimirimana; Claudine Ndoole; Priscilla Asiimwe; Angelique Kipenda; Phiona Nattabi; Joyeux Mugisho; Pole Pole).

Contributors Study design-CHL, M0, RH and LM. Data collection-RH, DK-M, $\mathrm{RL}, \mathrm{CHL}, \mathrm{MO}$ and IB. Data management- $\mathrm{BB}, \mathrm{CHL}$, DK-M, RH and RL. Manuscript writing - $\mathrm{BB}$ and $\mathrm{CHL}$. Manuscript editing and critical review- $\mathrm{CHL}, \mathrm{MO}, \mathrm{IB}, \mathrm{RH}, \mathrm{LM}$, DK-M, PK, SM, RL, AP-B and SB.

Funding This study is funded by the International Development Research Center (\# 109549-001). CHL is also funded by the Canada Research Chairs programme (\#Tie 2), Canada Foundation for Innovation (\#JELF), and the Ontario Ministry of Research and Innovation (ERA).

Disclaimer Funding agencies played no role in the design or execution of the study.

Competing interests RL is an academic physician-researcher and also has interests in a non-profit and private company social enterprise, WelTel, that develops and provides digital health software. He is not being paid or otherwise compensated by WelTel for this project. No other authors declare a conflict of interest.

Patient and public involvement Patients and/or the public were involved in the design, or conduct, or reporting, or dissemination plans of this research. Refer to the Methods section for further details.

\section{Patient consent for publication Not required}

Provenance and peer review Not commissioned; peer reviewed for ethical and funding approval prior to submission.

Open access This is an open access article distributed in accordance with the Creative Commons Attribution Non Commercial (CC BY-NC 4.0) license, which permits others to distribute, remix, adapt, build upon this work non-commercially, and license their derivative works on different terms, provided the original work is properly cited, appropriate credit is given, any changes made indicated, and the use is non-commercial. See: http://creativecommons.org/licenses/by-nc/4.0/.

\section{ORCID iDs}

Carmen H Logie http://orcid.org/0000-0002-8035-433X

Lawrence Mbuagbaw http://orcid.org/0000-0001-5855-5461

\section{REFERENCES}

1 Orcutt M, Patel P, Burns R, et al. Global call to action for inclusion of migrants and refugees in the COVID-19 response. The Lancet 2020;395:1482-3.

2 Orcutt M, Patel P, Burns R, et al. Global call to action for inclusion of migrants and refugees in the COVID-19 response. The Lancet 2020;395:1482-3. doi:10.1016/S0140-6736(20)30971-5

3 Singh L, Singh NS, Nezafat Maldonado B, et al. What does 'leave no one behind' mean for humanitarian crises-affected populations in the COVID-19 pandemic? BMJ Glob Health 2020;5:e002540. doi:10.1136/bmjgh-2020-002540

4 Kluge HHP, Jakab Z, Bartovic J, et al. Refugee and migrant health in the COVID-19 response. The Lancet 2020;395:1237-9. doi:10.1016/ S0140-6736(20)30791-1
5 Hargreaves S, Zenner D, Wickramage K, et al. Targeting COVID-19 interventions towards migrants in humanitarian settings. Lancet Infect Dis 2020;20:645-6. doi:10.1016/S1473-3099(20)30292-9

6 UNHCR. Global trends: forced displacement in 2020. Geneva, Switzerland: UNHCR, 2020. https://www.unhcr.org/60b638e37/ unhcr-global-trends-2020

7 Rees M. Foreword : time for cities to take centre stage on forced migration, 2020. Forced Migr Rev. Available: https://www.fmreview. org/cities/rees

8 Anzellini V, Leduc C. Urban internal displacement : data and evidence, 2020. Forced Migr Rev. Available: https://www.fmreview. $\mathrm{org} / \mathrm{cities} / \mathrm{anzellini-leduc}$

9 Sabila S, Silver I. Cities as partners: the case of Kampala, 2020. Forced Migr Rev. Available: https://www.fmreview.org/cities/salibasilver

10 UNHCR. Uganda - Refugee Statistics April 2021, 2021

11 Bukuluki P, Mwenyango H, Katongole SP, et al. The socio-economic and psychosocial impact of Covid-19 pandemic on urban refugees in Uganda. Soc Sci Humanit Open 2020;2:100045.

12 World Health Organization (WHO). Coronavirus disease (COVID-10) advice for the public, 2021. Available: https://www.who.int/ emergencies/diseases/novel-coronavirus-2019/advice-for-public

13 Singh NS, Abrahim O, Altare C, et al. COVID-19 in humanitarian settings: documenting and sharing context-specific programmatic experiences. Confl Health 2020;14:79.

14 Nasreen S, Azziz-Baumgartner E, Gurley ES, et al. Prevalent high-risk respiratory hygiene practices in urban and rural Bangladesh. Trop Med Int Heal 2010;15:762-71.

15 Sultana F, Nizame FA, Southern DL, et al. Pilot of an elementary school cough etiquette intervention: acceptability, feasibility, and potential for sustainability. Am J Trop Med Hyg 2017;97:1876-85.

16 Stebbins S, Cummings DAT, Stark JH, et al. Reduction in the incidence of influenza A but not influenza B associated with use of hand sanitizer and cough hygiene in schools: a randomized controlled trial. Pediatr Infect Dis J 2011;30:921-6.

17 Phillips RM, Vujcic J, Boscoe A, et al. Soap is not enough handwashing practices and knowledge in refugee camps, Maban County, South Sudan. Confl Health 2015;9:39.

18 Vujcic J, Ram PK, Blum LS. Handwashing promotion in humanitarian emergencies: strategies and challenges according to experts. $J$ Water, Sanit Hyg Dev 2015;5:574-85.

19 Watson J, Cumming O, Aunger R, et al. Child handwashing in an internally displaced persons CAMP in northern Iraq: a qualitative multi-method exploration of motivational drivers and other handwashing determinants. PLoS One 2020;15:e0228482.

20 Blum LS, Yemweni A, Trinies V, et al. Programmatic implications for promotion of handwashing behavior in an internally displaced persons cAMP in North Kivu, Democratic Republic of Congo. Confl Health 2019;13:54.

21 Subbaraman N. 'Distancing is impossible': refugee camps race to avert coronavirus catastrophe. Nature 2020 https://www.nature.com/ articles/d41586-020-01219-6

22 Ramalingam B, Singh NS, Mahieu A, et al. Responding to COVID-19: guidance for humanitarian agencies. London: ODI/ALNAP, 2020.

23 Behavioural science must be at the heart of the public health response to covid-19, 2020. The BMJ. Available: https://blogs.bmj. $\mathrm{com} / \mathrm{bmj} / 2020 / 02 / 28 /$ behavioural-science-must-be-at-the-heart-ofthe-public-health-response-to-covid-19/

24 Mosler H-J. A systematic approach to behavior change interventions for the water and sanitation sector in developing countries: a conceptual model, a review, and a guideline. Int J Environ Health Res 2012;22:431-49.

25 Contzen N, Mosler HJ. The RANAS approach to systematic behavior change: methodological fact sheet 1. Dübendorf, Switzerland: Eawag, Swiss Federal Institute of Aquatic Science and Technology, 2015.

26 Graf J, Meierhofer R, Wegelin M, et al. Water disinfection and hygiene behaviour in an urban slum in Kenya: impact on childhood diarrhoea and influence of beliefs. Int J Environ Health Res 2008;18:335-55. doi:10.1080/09603120801966050

27 Contzen N, Meili IH, Mosler H-J. Changing handwashing behaviour in southern Ethiopia: a longitudinal study on infrastructural and commitment interventions. Soc Sci Med 2015;124:103-14. doi:10.1016/j.socscimed.2014.11.006

28 Contzen N, Mosler H-J. Impact of different promotional channels on handwashing behaviour in an emergency context: Haiti postearthquake public health promotions and cholera response. J Public Health 2013;21:559-73. doi:10.1007/s10389-013-0577-4

29 Friedrich MND, Binkert ME, Mosler H-J. Contextual and psychosocial determinants of effective handwashing technique: recommendations for interventions from a case study in Harare, 
Zimbabwe. Am J Trop Med Hyg 2017;96:430-6. doi:10.4269/ ajtmh.16-0553

30 WhatsApp Blog. Connecting one billion users every day, 2017. Available: https://blog.whatsapp.com/connecting-one-billion-usersevery-day

31 Legido-Quigley H, Asgari N, Teo YY, et al. Are high-performing health systems resilient against the COVID-19 epidemic? Lancet 2020;395:848-50. doi:10.1016/S0140-6736(20)30551-1

32 Zotti F, Dalessandri D, Salgarello S, et al. Usefulness of an app in improving oral hygiene compliance in adolescent orthodontic patients. Angle Orthod 2016;86:101-7. doi:10.2319/010915-19.1

33 Cheung YTD, Chan CHH, Lai C-KJ, et al. Using WhatsApp and Facebook online social groups for smoking relapse prevention for recent quitters: a pilot pragmatic cluster randomized controlled trial. J Med Internet Res 2015;17:e238. doi:10.2196/jmir.4829

34 Alanzi TM, Bah S, Jaber F. Evaluation of a mobile social networking application for glycaemic control and diabetes knowledge in patients with type 2 diabetes: a randomized controlled trial using WhatsApp, 2016. Qatar Foundation annual research conference proceedings. Hamad bin Khalifa university press. Available: https://www.qscience. com/content/papers/10.5339/qfarc.2016.HBPP2533

35 Lester RT, Ritvo P, Mills EJ, et al. Effects of a mobile phone short message service on antiretroviral treatment adherence in Kenya (WelTel Kenya1): a randomised trial. Lancet 2010;376:1838-45.

36 van der Kop ML, Muhula S, Nagide PI, et al. Effect of an interactive text-messaging service on patient retention during the first year of HIV care in Kenya (WelTel retain): an open-label, randomised parallelgroup study. Lancet Public Health 2018;3:e143-52.

37 UN Refugee Agency. Connecting refugees: how Internet and mobile connectivity can improve refugee well-being and transform humanitarian action, 2016. Available: http://www.unhcr.org/ connectivity-for-refugees.htm

38 Logie C, Okumu M, Hakiza R, et al. Mobile health-supported HIV self-testing strategy among urban refugee and displaced youth in Kampala, Uganda: protocol for a cluster randomized trial (Tushirikiane, supporting each other). JMIR Res Protoc 2021;10:e26192.

39 Smith M, Upshur R, Disease P. Pandemic disease, public health, and ethics. In: The Oxford Handbook of public health ethics, 2019.

40 Campbell AR, Kinvig K, Côté HC, et al. Health care provider utilization and cost of an mHealth intervention in vulnerable people living with HIV in Vancouver, Canada: prospective study. JMIR Mhealth Uhealth 2018;6:e152. doi:10.2196/mhealth.9493

41 Rouhani SA, Marsh RH, Rimpel L, et al. Social messaging for global health: lessons from Haiti. J Glob Health 2019;9:010308. doi:10.7189/jogh.09.010308

42 Shen C, Wang MP, Wan A, et al. Health information exposure from information and communication technologies and its associations with health behaviors: population-based survey. Prev Med 2018;113:140-6. doi:10.1016/j.ypmed.2018.05.018

43 Dorwal P, Sachdev R, Gautam D, et al. Role of WhatsApp messenger in the laboratory management system: a boon to communication. J Med Syst 2016;40:14. doi:10.1007/s10916-0150384-2

44 Ellanti P, Moriarty A, Coughlan F, et al. The use of WhatsApp smartphone messaging improves communication efficiency within an orthopaedic surgery team. Cureus 2017;9:e1040. doi:10.7759/ cureus. 1040

45 Gamma AE, Slekiene J, von Medeazza G, et al. Contextual and psychosocial factors predicting Ebola prevention behaviours using the RANAS approach to behaviour change in Guinea-Bissau. BMC Public Health 2017;17:446.

46 Kroenke K, Spitzer RL, Williams JB. The PHQ-9: validity of a brief depression severity measure. J Gen Intern Med 2001;16:606-13.

47 Des Jarlais DC, Lyles C, Crepaz N, et al. Improving the reporting quality of nonrandomized evaluations of behavioral and public health interventions: the trend statement. Am J Public Health 2004;94:361-6. doi:10.2105/AJPH.94.3.361 